PROCEEDINGS OF THE

AMERICAN MATHEMATICAL SOCIETY

Volume 126, Number 4, April 1998, Pages 1109-1113

S 0002-9939(98)04233-6

\title{
MAXIMAL NESTS IN THE CALKIN ALGEBRA
}

\author{
DON HADWIN
}

(Communicated by Palle E. T. Jorgensen)

\begin{abstract}
We prove that if two countable commutative lattices of projections in the Calkin algebra are order isomorphic, then they are unitarily equivalent. We show that there are isomorphic maximal nests of projections in the Calkin algebra that are order isomorphic but not similar. Assuming the continuum hypothesis, we show that all maximal nests of projections in the Calkin algebra are order isomorphic.
\end{abstract}

Suppose $H$ is a separable infinite-dimensional Hilbert space, let $B(H)$ denote the algebra of all operators on $H$, and let $\mathcal{K}(H)$ denote the ideal of compact operators on $H$. We let $\mathcal{C}(H)$ denote the Calkin algebra, $B(H) / \mathcal{K}(H)$.

A nest in $B(H)$ is a linearly ordered family of projections containing 0 and 1 . We identify projections in $B(H)$ with their ranges in $H$ (i.e., each subspace of $H$ corresponds to the orthogonal projection onto that subspace). By a Boolean algebra of projections, we mean a commutative family $\mathcal{B}$ of projections containing 0 and 1 that is a Boolean algebra under $P \vee Q=P+Q-P Q, P \wedge Q=P Q$, and $P^{\prime}=1-P$.

Two nests $\mathcal{P}$ and $\mathcal{Q}$ are similar if there is an invertible operator $S$ such that $\mathcal{Q}=\{\operatorname{ran} S P: P \in \mathcal{P}\}$. Note that the projection onto $\operatorname{ran} S P$ can be written as $f\left(\left(S P S^{-1}\right)\left(S P S^{-1}\right)^{*}\right)$, where $f:[0, \infty) \rightarrow[0,1]$ is the continuous function $f(t)=$ $\min \{1, t\}$. The question of similarity of nests of subspaces of a separable infinitedimensional Hilbert space goes back to J. Ringrose, who asked if similar nests must be unitarily equivalent. Using a result of N. T. Andersen [A] (see also [Ar]), D. Larson [L] gave a negative answer to Ringrose's question. Building on Larson's ideas, K. Davidson [D1] gave the following wonderful characterization of similarity for nests. A beautiful account of these facts is contained in [D2].

Theorem 1. [D1] Suppose $\mathcal{P}$ and $\mathcal{Q}$ are nests in $B(H)$ that are closed in the strong operator topology. Then $\mathcal{P}$ is similar to $\mathcal{Q}$ if and only if there is an order isomorphism $\tau: \mathcal{P} \rightarrow \mathcal{Q}$ such that, for every $P, Q \in \mathcal{P}$ with $P<Q$, $\operatorname{rank}(Q-P)=$ $\operatorname{rank}(\tau(Q)-\tau(P))$. Moreover, given $\tau$ and $\epsilon>0$, there exist a unitary operator $U$ and a compact operator $K$ with $\|K\|<\epsilon$ such that $S=U+K$ is invertible and $\tau(P)=\operatorname{ran} S P$ for every $P$ in $\mathcal{P}$.

Note that the preceding theorem shows that rank-preserving order isomorphisms of nests in $B(H)$ are given by a unitary operator in $\mathcal{C}(H)$. This note addresses the question of what analogs of these aforementioned results hold in the Calkin algebra $\mathcal{C}(H)$. Similar problems were studied in [AD] and [AG]. Since there is no analog of finite-rank projections in $\mathcal{C}(H)$, one might suspect that any two order

Received by the editors September 23, 1996.

1991 Mathematics Subject Classification. Primary 47D25, 04 A30. 
isomorphisms between nests are implemented by a similarity. Indeed, we show that order isomorphisms between countable nests (or even commutative lattices) are unitarily implemented. However, we show that, given a maximal nest $\mathcal{M}$ in $\mathcal{C}(H)$, there are $2^{c}$ distinct maximal nests in $\mathcal{C}(H)$ that are order isomorphic to $\mathcal{M}$. Hence, since $\operatorname{cardC}(H)=c$, there is a maximal nest that is order isomorphic to $\mathcal{M}$ that is not similar to $\mathcal{M}$ or even representable in terms of a sequence of similarities. The question of whether all maximal nests in $\mathcal{C}(H)$ are order isomorphic may depend on the continuum hypothesis $(\mathrm{CH})$, the assertion that $\aleph_{1}=c$, where $c=2^{\aleph_{0}}$ is the cardinality of the continuum. Assuming $(\mathrm{CH})$, we show that every two maximal nests in the Calkin algebra are order isomorphic, and that each such nest has $2^{c}$ distinct order-automorphisms, so they cannot all be defined even in terms of sequences of similarities (there are only $c$ of them). Note that, although the set of projections in the Calkin algebra does not form a lattice, a commutative family of projections in a $C^{*}$-algebra generates a lattice with the operations $p \wedge q=p q$ and $p \vee q=p+q-p q$. We call a lattice of commuting projections a commutative lattice.

Theorem 2. Suppose $H$ is a separable infinite-dimensional Hilbert space; $\mathcal{B}_{1}$ and $\mathcal{B}_{2}$ are countable commutative lattices of projections in $\mathcal{C}(H)$ containing 0 and 1. Suppose $\tau: \mathcal{B}_{1} \rightarrow \mathcal{B}_{2}$ is an order isomorphism. Then there is a unitary operator $U$ in $H$ with image $u$ in $\mathcal{C}(H)$, such that, for every $p$ in $\mathcal{B}_{1}, \tau(p)=u^{*} p u$.

Proof. Suppose $\mathcal{B}_{1}=\left\{0,1, p_{1}, p_{2}, \ldots\right\}$ and $\mathcal{B}_{2}=\left\{0,1, \tau\left(p_{1}\right), \tau\left(p_{2}\right), \ldots\right\}$. It is clear, for each positive integer $n$, that $\tau$ extends uniquely to an isomorphism between the Boolean algebras generated by $\left\{0,1, p_{1}, \ldots, p_{n}\right\}$ and $\left\{0,1, \tau\left(p_{1}\right), \ldots, \tau\left(p_{n}\right)\right\}$. Hence we can assume that $\mathcal{B}_{1}$ and $\mathcal{B}_{2}$ are Boolean algebras. It follows that $\tau$ extends to a unital ${ }^{*}$-isomorphism, which we still call $\tau$, from $C^{*}\left(\mathcal{B}_{1}\right)$ onto $C^{*}\left(\mathcal{B}_{2}\right)$. However, by [R, p. 293], $C^{*}\left(\mathcal{B}_{1}\right)$ is generated by a single hermitian element $h$ (e.g., $h=$ $\left.\sum_{k=1}^{\infty} p_{k} / 3^{k}\right)$. Then $C^{*}\left(\mathcal{B}_{2}\right)$ is generated by $\tau(h)$. Hence $\sigma(h)=\sigma(\tau(h))$, and it follows from the Weyl-von Neumann theorem [vN1] that there is a unitary operator $U$ in $B(H)$ whose image in $\mathcal{C}(H)$ is $u$ such that $\tau(h)=u^{*} h u$. It follows that $\tau(x)=u^{*} x u$ for every $x$ in $C^{*}(a)$. Whence, $\tau\left(p_{n}\right)=u^{*} p_{n} u$ for $n=1,2, \ldots$

Suppose $m$ is a cardinal. We say that a partially ordered set $(X, \leq)$ is $m$-saturated if for each pair $A, B \subset X$ with $\operatorname{card} A, \operatorname{card} B \leq m$ and $A<B$ (i.e., $a<b$ for all $a \in A$ and $b \in B$ ), there is an $x$ in $X$ such that $A<\{x\}<B$. In $1908 \mathrm{~F}$. Hausdorff [H] proved that if $\mathrm{k}$ is an infinite cardinal and $(X, \leq),(Y, \leq)$ are two linearly ordered sets, each with a largest and smallest element, such that, for every cardinal $m<k$, $X$ and $Y$ are both $m$-saturated, then $X$ and $Y$ are order isomorphic. Hausdorff's proof, which involves a "back-and-forth" argument, also yields the existence of $k^{k}$ distinct order isomorphisms between $X$ and $Y$. Most likely the reader will see the idea of Hausdorff's proof by proving that the set of rationals in $[0,1]$ is order isomorphic with the set of dyadic rationals in $[0,1]$. For readable accounts of these ideas, consult $[\mathrm{CN}]$ and $[\mathrm{DW}]$.

Lemma 3. If $\mathcal{M}$ is a maximal projection chain in $\mathcal{C}(H)$ or $\ell^{\infty} / c_{o}$, then $\mathcal{M}$ is $\aleph_{0}$-saturated.

Proof. We give the proof for $\mathcal{C}(H)$. Suppose $\left\{p_{n}\right\}$ and $\left\{q_{n}\right\}$ are sequences of projections in $\mathcal{C}(H)$ such that $p_{1}<p_{2}<\cdots<\cdots<q_{2}<q_{1}$. We can find sequences $\left\{P_{n}\right\}$ and $\left\{Q_{n}\right\}$ of projections in $B(H)$ such that, for each $n, p_{n}$ is the image in $\mathcal{C}(H)$ of $P_{n}$ and $q_{n}$ is the image in $\mathcal{C}(H)$ of $Q_{n}$, and $P_{1}<P_{2}<\cdots<\cdots<Q_{2}<Q_{1}$. For 
each $n$, we can choose a unit vector $e_{n}$ in $\operatorname{ran}\left(P_{n+1}-P_{n}\right)$, and define $\widetilde{P}_{n+1}$ to be the projection onto $P_{n+1}(H) \cap\left\{e_{1}, \ldots, e_{n}\right\}^{\perp}$. Let $P$ be the supremum of the $\widetilde{P}_{n+1}$ 's plus the projection onto the closed linear span of $\left\{e_{1}, e_{3}, e_{5}, e_{7}, \ldots\right\}$. Let $p$ be the image of $P$ in $\mathcal{C}(H)$. Then $p_{1}<p_{2}<\ldots<p<\ldots<q_{2}<q_{1}$.

Now suppose $\mathcal{M}$ is a maximal chain of projections in $\mathcal{C}(H)$. Suppose $A$ and $B$ are countable subsets of $\mathcal{M}$ such that $p<q$ for every $p$ in $A$ and every $q$ in $B$. We can assume that $A$ is infinite (if $B$ is infinite, look at the chain $\{1-p: p \in \mathcal{M}\}$ ). The argument in the preceding paragraph shows that there is a projection $p^{\prime}$ in $\mathcal{C}(H)$ such that $p<p^{\prime}<q$ for every $p$ in $A$ and every $q$ in $B$. If there is no projection $q^{\prime}$ in $\mathcal{M}$ such that $p<q^{\prime}<q$ for every $p$ in $A$ and every $q$ in $B$, then $\mathcal{M} \cup\left\{p^{\prime}\right\}$ would violate the maximality of $\mathcal{M}$. Hence $\mathcal{M}$ is $\aleph_{0}$-saturated

Theorem 4. Assume the continuum hypothesis holds. Then any two maximal nests of projections in $\mathcal{C}(H)$ are order isomorphic. Moreover, any maximal nest in $\mathcal{C}(H)$ has $2^{c}$ order-automorphisms.

Corollary 5. Assuming the continuum hypothesis, there exist a nest and an orderautomorphism on it that is not implemented by a similarity, or even a sequence of similarities.

Conjecture. If $(\mathrm{CH})$ fails, then there are two maximal nests in $\mathcal{C}(H)$ that are not order isomorphic.

Example. Hausdorff $[\mathrm{H}]$ also proved that every pair of $\aleph_{0}$-saturated Boolean algebras having cardinality $\aleph_{1}$ are isomorphic. However, neither Lemma 3 nor Theorem 4 is true for maximal Boolean algebras of commuting projections in the Calkin algebra. For example, let $\mathcal{A}$ be the algebra of all diagonal operators with respect to a fixed countable orthonormal basis for the Hilbert space $H$, and suppose $\mathcal{B}$ is unitarily equivalent to $L^{\infty}(\mu)$ acting on $L^{2}(\mu)$, where $\mu$ is Lebesgue measure on $[0,1]$. Let $\mathcal{P}$ be the image in the Calkin algebra of the projections in $\mathcal{A}$ and let $\mathcal{Q}$ be the image in the Calkin algebra of the projections in $\mathcal{B}$. It follows from a theorem of B. Johnson and S. K. Parrott [JP] that $\mathcal{P}$ and $\mathcal{Q}$ are maximal Boolean algebras of projections in $B(H) / \mathcal{K}(H)$, i.e., $C^{*}(\mathcal{P})$ is the image of $\mathcal{A}$ in $B(H) / \mathcal{K}(H)$ and is a masa in $B(H) / \mathcal{K}(H)$. However, $\mathcal{B} \cap \mathcal{K}(H)=\{0\}$, and thus $\mathcal{Q}$ contains no $\aleph_{0}$-saturated chain. However, by Lemma $3, \mathcal{P}$ does contain such a chain. Hence $\mathcal{P}$ and $\mathcal{Q}$ are not isomorphic.

While Corollary 5 shows that not every order isomorphism between maximal nests in $\mathcal{C}(H)$ is induced by a similarity, it leaves open the question of whether order isomorphic nests in $\mathcal{C}(H)$ are similar. The next result provides a negative answer.

Theorem 6. Suppose $\mathcal{M}$ is a maximal nest in $\mathcal{C}(H)$. Then there are at least $2^{c}$ distinct maximal nests in $\mathcal{C}(H)$ that are order isomorphic to $\mathcal{M}$.

Proof. Let $\mathbb{Q}$ denote the rational numbers in $[0,1]$. It is clear that $\mathbb{Q}$ is order isomorphic to a subset $\left\{p_{t}: t \in \mathbb{Q}\right\}$ of $\mathcal{M}$. For each irrational number $t$ in $[0,1]$, we can apply Lemma 3 to obtain a projection $p_{t}$ such that for every $r, s$ in $\mathbb{Q}$ with $r<t<s$, we have $p_{r}<p_{t}<p_{s}$. Hence $\left\{p_{t}: t \in[0,1]\right\}$ is order isomorphic to $[0,1]$.

Suppose $E \subset(0,1)$. By Lemma 3 and the fact that $\mathbb{Q}$ is order-dense in $[0,1]$, we can, for each $t$ in $E$, find projections $p_{t^{+}}$and $p_{t^{-}}$in $\mathcal{M}$ such that $p_{s}<p_{t^{-}}<p_{t}<$ $p_{t^{+}}<p_{r}$ holds in $\mathcal{C}(H)$ whenever $0 \leq s<t<r \leq 1$. Clearly $p_{t^{+}}<p_{r^{-}}$holds when $t, r \in E$ and $t<r$. 
For each $t$ in $E$, choose projections $P_{t^{-}}, P_{t}, P_{t^{+}}$in $B(H)$ such that $P_{t^{-}}<P_{t}<$ $P_{t^{+}}$and the images of $P_{t^{-}}, P_{t}, P_{t^{+}}$, respectively, are $p_{t^{-}}, p_{t}, p_{t^{+}}$. It follows that

$$
\left\{p-p_{t^{-}}: p \in \mathcal{M}, p_{t^{-}} \leq p \leq p_{t^{+}}\right\}
$$

can be represented as a maximal nest in $\mathcal{C}\left(\left[P_{t^{+}}-P_{t^{-}}\right](H)\right)$, which can in turn be represented by a family $\mathcal{P}_{t}$ of projections in $B\left(\left[P_{t^{+}}-P_{t^{-}}\right](H)\right)$. We can choose a projection $Q_{t}$ and a unitary operator $U_{t}$ in $B\left(\left[P_{t^{+}}-P_{t^{-}}\right](H)\right)$ such that $Q_{t}\left(P_{t}-P_{t^{-}}\right)-$ $\left(P_{t}-P_{t^{-}}\right) Q_{t}$ is not compact and such that $U_{t} Q_{t}-\left(P_{t}-P_{t^{-}}\right) U_{t}=0$. We now define a maximal chain $\mathcal{M}_{E}$ to be the union of $\left\{p \in \mathcal{M}\right.$ : there is no t in $E$ with $\left.p_{t^{-}} \leq p \leq p_{t^{+}}\right\}$ with the union over $t \in E$ of the image in $\mathcal{C}(H)$ of $\left\{P_{t^{-}}+U_{t} P U_{t}{ }^{*}: P \in \mathcal{P}_{t}\right\}$. It is clear that $\mathcal{M}_{E}$ is order isomorphic to $\mathcal{M}$ and that $\left\{t \in(0,1): p_{t} \in \mathcal{M}_{E}\right\}=E$.

It follows that there are at least as many maximal nests in $\mathcal{C}(H)$ isomorphic to $\mathcal{M}$ as there are subsets $E$ of $(0,1)$, namely, $2^{c}$.

Corollary 7. For each maximal nest $\mathcal{M}$ in $\mathcal{C}(H)$ there is a maximal nest in $\mathcal{C}(H)$ that is order isomorphic to $\mathcal{M}$ but not similar to $\mathcal{M}$.

We conclude by noting that Ringrose's original question remains unanswered in the Calkin algebra.

Question. Are two similar nests of projections in the Calkin algebra unitarily equivalent?

\section{ACKNOWLEDGMENT}

The author was supported by a research grant from the National Science Foundation while this research was undertaken.

\section{REFERENCES}

[A] N. T. Andersen, Similarity of continuous nests, Bull. London Math. Soc. 15 (1983) 131-132. MR 85b: 47049

[AD] C. Apostol and K. R. Davidson, Isomorphisms modulo the compact operators of nest algebras, II, Duke Math. J. 6 (1988) no. 1, 101-127. MR 89g:47058

[AG] C. Apostol and F. Gilfeather, Isomorphisms modulo the compact operators of nest algebras, Pacific J. Math. 122 (1986) 263-286. MR 87f:47063

[Ar] W. B. Arveson, Perturbation theory for groups and lattices, J. Funct. Anal. 53 (1983) 22-73. MR 85d:47042

[BDF] L. Brown, R. G. Douglas, and P. A. Fillmore, BDF

$[\mathrm{CN}]$ W. W. Comfort and S. Negrepontis, The theory of ultrafilters, Springer-Verlag, Berlin, 1974. MR 53:135

[DW] H. G. Dales and W. H. Woodin, An introduction to independence for analysts, London Math. Soc. Lecture Notes, No. 115, Cambridge Univ. Press, 1987. MR 90d:03101

[D1] K. R. Davidson, Similarity and compact perturbations of nest algebras, J. Reine Angew. Math. 348 (1984), 286-294. MR 86c:47062

[D2] K. Davidson, Nest algebras, Pitman Res. Notes Math. Ser., no. 191, Longman Sci. Tech., Harlow, 1988. MR 90f: 47062

[H] F. Hausdorff, Summen von $\aleph_{1}$ Mengen, Fund. Math. 26 (1936) 241-255.

[JP] B. E. Johnson and S. K. Parrott, Operators commuting with von Neumann algebras modulo the set of compact operators, J. Funct. Anal. 11 (1972) 39-61. MR 49:5869

[L] D. R. Larson, A solution to a problem of J. R. Ringrose, Bull. Amer. Math. Soc. 7 (1982) 243-246. MR 83k:46046 
[R] C. A. Rickart, General theory of Banach algebras, Van Nostrand, Princeton, 1960. MR 22:5903

[vN1] J. von Neumann, Charakterisierung des Spektrums eines Integral Operators, Hermann, Paris, 1935.

Department of Mathematics, University of New Hampshire, Durham, New Hampshire 03824

E-mail address: don@math.unh.edu 